\title{
Airway-associated macrophages in homeostasis and repair
}

Anna E. Engler ${ }^{1}$, Alexandra B. Ysasi ${ }^{1}$, Riley M.F. Pihl ${ }^{2}$, Carlos Villacorta-Martin ${ }^{1}$, Hailey M. Heston1, Hanne M.K. Richardson¹, Noah R. Moniz¹, Anna C. Belkina2,3, Sarah A. Mazzilli4 , Jason R. Rock1, *

${ }^{1}$ Center for Regenerative Medicine, Boston University School of Medicine, Boston, MA, 02118, USA

2 Flow Cytometry Core Facility, Boston University School of Medicine, Boston, MA, 02118, USA

3 Department of Pathology and Laboratory Medicine, Boston University School of Medicine, Boston, MA, 02118, USA

4 Department of Medicine and Section of Computational Biomedicine, Boston University School of Medicine, Boston, MA, 02118, USA.

* corresponding author: rockjr@bu.edu

\section{Summary}

There is an increasing appreciation for the heterogeneity of myeloid lineages in the respiratory system, but whether distinct populations associate with the conducting airways remains unknown. We use single cell RNA sequencing, flow cytometry and immunofluorescence to characterize myeloid cells of the mouse trachea during homeostasis and epithelial injury/repair. We identify submucosal macrophages that are similar to lung interstitial macrophages and intraepithelial macrophages, and find that repair of the tracheal epithelium is impaired in Ccr2-deficient mice. Following injury there are early increases in neutrophils and submucosal macrophages, including M2-like macrophages. Unexpectedly, intraepithelial macrophages are initially lost but later replaced from $\mathrm{CCR}^{+}$monocytes. Mast cells and group 2 innate lymphoid cells are sources of IL13 that polarizes macrophages and directly influences basal cell behaviors. Their proximity to the airway epithelium establishes these myeloid populations as potential therapeutic targets for airway disease. 


\section{Introduction}

The respiratory system can be divided into at least two functional zones - the proximal conducting airways and the distal alveoli - and each contains distinct populations of epithelial and mesenchymal cells with unique functions (Rock and Hogan, 2011). Alveolar macrophages (AMs) are thought to be the tissue resident macrophages of the lung, and there is evidence for ontological, molecular and functional heterogeneity in this population (Misharin et al., 2013; Tan and Krasnow, 2016). Much less is known about the myeloid lineages of the airways, but recent evidence points to molecularly distinct interstitial macrophages (IMs) associated with the submucosa (Gibbings et al., 2017). We have exploited the mouse trachea as a model for smaller airways, including those of humans (Rock et al., 2010) to learn more about these cells during homeostasis and repair after injury.

Recent studies have demonstrated roles for immune modulation of lung repair and regeneration. We recently demonstrated that $\mathrm{CCR} 2^{+}$inflammatory monocytes and M2like $\mathrm{Arg} 1^{+}$macrophages promote regeneration from type 2 alveolar epithelial stem cells in a mouse model of partial pneumonectomy (Lechner et al., 2017). In the airways, it is well known that Th2 cytokines influence the cellular composition of the epithelium (Danahay et al., 2015; Kuperman et al., 2002), but less is known about how myeloid lineages impact injury and repair. To address this question, we used flow cytometry and single cell RNA sequencing ( $\mathrm{SCSeq}$ ) to characterize the myeloid populations in the mouse trachea at steady state and during repair of the epithelium following chemicallyinduced injury, when the number of macrophages increases (Tadokoro et al., 2014). We provide evidence for airway-associated macrophages that are distinct from AMs and show that their polarization shifts from M0-like to M2-like during epithelial repair. These M2-like macrophages originate, at least in part, from a $\mathrm{Ccr}^{+}$precursor and regeneration of the tracheal epithelium is impaired in the absence of Ccr2 expression. Lastly, we identify a unique macrophage subset that is located in close proximity to airway basal cells within the tracheal epithelium. Our data suggest that these cells are lost along with 
the tracheal epithelium after chemically-induced injury and are replaced by CCR2+ cells during epithelial repair.

\section{Results}

The trachea contains interstitial macrophages under homeostatic conditions

The mouse trachea provides a well-characterized model of the pseudostratified airway epithelium. To characterize the macrophages associated with this tissue we first performed flow cytometry on cells from the dissociated normal trachea and from the remaining lung lobes for comparison. This identified a population of $\mathrm{CD} 45^{+} ; \mathrm{F} 4 / 80^{+}$ myeloid cells in the trachea which are negative for SiglecF (Figure S1A-B), a classical marker of alveolar macrophages (AMs). Approximately $38 \%$ of the CD45+ cells in the trachea are MerTK+. Most of these are CD11 bhi and variably express CD11C (FigureS1B), a phenotype consistent with interstitial macrophages (IM) previously described for the

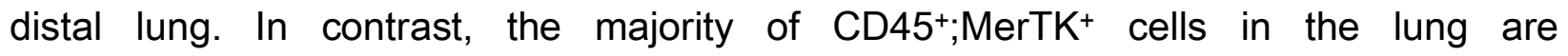

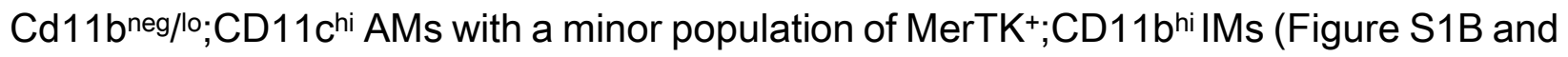
(Gibbings et al., 2017)).

\section{Tracheal macrophage dynamics during injury and repair of the tracheal epithelium}

Following infection, IMs expand through local proliferation and modulate lung inflammation (Kawano et al., 2016; Ural et al., 2020). We used a model of polidocanolinduced injury to determine how the tracheal macrophage populations respond to noninfectious injury of the airway epithelium (Borthwick et al., 2001). We analyzed the tracheas of polidocanol injured and PBS treated sham control animals 1-, 3- and 7-days post injury (hereafter $1 \mathrm{dpP}, 3 \mathrm{dpP}$ and $7 \mathrm{dpP}$ ) (Figure S1C-D). Polidocanol results in the death and sloughing of suprabasal ciliated and secretory cells of the pseudostratified epithelium within 3 hours, largely sparing basal cells (Figure S1D). The normal proportions of epithelial cells and epithelial morphology are restored within 1-2 weeks (Figure S1D) by the proliferation and differentiation of basal cells (Borthwick et al., 2001; Plasschaert et al., 2018)). At $1 \mathrm{dpP}$ we observed an increase in the number of CD45+ 
cells in the mesenchyme beneath the injured epithelium (Figure 1A). Flow cytometry showed a rapid increase of $\mathrm{CD} 45^{+}$cells and $\mathrm{CD} 45^{+} \mathrm{F} 4 / 80^{+}$cells within $1 \mathrm{dpP}$ (Figure 1B). We then carried out a more detailed analysis using a 15-color myeloid immunophenotyping panel (Supplemental Table S1). Opt-SNE dimensionality reduction and PhenoGraph unsupervised clustering of live, extravascular CD45+ cells from sham and 1, 3 and $7 \mathrm{dpP}$ tracheas showed 24 distinct clusters (Figure 1C, S1E), of which 17 were categorized as myeloid populations (Figure 1D). The proportion of cells expressing a cell surface phenotype consistent with neutrophils rapidly increased $1 \mathrm{dpP}$ compared to controls (Fig 1C and E, Clusters 10, 2 and 3). We also observed increases in the numbers of $\mathrm{Ly} 6 \mathrm{C}^{+}$putative newly recruited macrophages (Cluster 9) and tissue macrophages (Cluster 4 ) at $3 \mathrm{dpP}$. Within $7 \mathrm{dpP}$, cellular composition had returned to baseline (Figure 1E-F). These data reveal dynamic changes in the myeloid populations during repair and implicate monocyte recruitment as a potential contributing mechanism.

\section{Transcriptional changes during injury and repair}

To further characterize the immune composition during tracheal injury and repair, we performed SCSeq of $\mathrm{CD} 45^{+}$cells from dissociated tracheas of uninjured control and mice 1, 4 and $7 \mathrm{dpP}$. We performed post-processing filtering to focus our analyses on myeloid cells $\left(\mathrm{F} 4 / 80^{+}\right.$, Adgre $\left.1^{+}\right)$(Figure $\left.2 \mathrm{~A}, \mathrm{~S} 2 \mathrm{~A}\right)$. Uniform manifold approximation and projection (UMAP) visualization (Figure 2B) and analysis of differential gene expression (Figure S2B, Supplemental Table S2) shows that the myeloid cells from uninjured tracheas and 4 and $7 \mathrm{dpP}$ are largely overlapping and these are enriched for classic macrophage and monocyte signatures (Figure $2 \mathrm{C}$-D). There were also clusters of cells with signatures of dendritic cells (DCs) $\left(\mathrm{Cd}_{103^{+}}\right.$; $\left.\mathrm{Cd} 11 \mathrm{~b}^{+}\right)$, presumably expressing low levels of Adgre1 detectable by sequencing but not FACS. Upon injury, the number of cells expressing transcripts associated with M2-like macrophages, such as Arg1 and Arg2 are increased (Figure 2C, Figure S2B, Supplemental Table S2). Concomitantly the expression of transcripts associated with $\mathrm{CCr}^{+}$and $\mathrm{C} \times 3 \mathrm{Cr}^{1+}$ monocytes is rapidly reduced, but returns to baseline $4 \mathrm{dpP}$ and $7 \mathrm{dpP}$ (Figure 2D). We did not observe significant changes in 
transcripts associated with cell cycle during the course of injury and repair (Figure 2E, gene set Supplemental Table S4).

\section{The number of M2-like macrophages increases following polidocanol-induced injury}

We performed flow cytometry to confirm our observations on the protein level. Consistent with earlier data (Figure 1), the number of $\mathrm{CD} 45^{+} ; \mathrm{F} 4 / 80^{+}$myeloid cells increased at 1 and $3 \mathrm{dpP}$ and returned to baseline by $7 \mathrm{dpP}$ (Figure S2C). We used an Arginase-YFP reporter mouse (YARG, (Reese et al., 2007)) to quantify M2-like macrophages in the trachea by flow cytometry. YARG ${ }^{+}$cells were uniquely increased at $3 \mathrm{dpP}$ compared to sham and other intervals post-injury (Figure 2F, S2C). This translational readout of arginase expression increased slightly later than the M2-like macrophage associated transcripts (Figure 2B).

Because flow cytometry demonstrated an increase in newly recruited monocytes and macrophages 1-3 dpP (Figure 1C-F), we asked whether the M2-like macrophages were derived from a CCR2+ monocyte precursor. We administered tamoxifen to Ccr2CreER ${ }^{\mathrm{T} 2} ; \mathrm{ROSA}$-tdTom;YARG mice to lineage trace $\mathrm{CCR}^{+}$monocytes and determine whether they gave rise to $\mathrm{YARG}^{+}$cells (Figure S2D). Two weeks later we injured the tracheal epithelium with polidocanol and analyzed tracheas at 3dpP (Figure S2D). As above, the number of CD45+ cells was increased $3 \mathrm{dpP}$ compared to sham. At this time the percentage of CD45+ cells that are Tom ${ }^{+}$cells decreased (Figure 2G), but the total number of Tom+ cells was unchanged (Sham: 3018 +/- 1549; Polidocanol: 2717 +/- 706;). This result presumably reflects the local proliferation and/or recruitment of CCR2-negative leukocytes, including neutrophils, lymphocytes and other monocyte subpopulations (Figure 1 and (Joshi et al., 2020; Ural et al., 2020)). Consistent with our transcription and flow cytometry data, both the total number and proportion of $\mathrm{YARG}^{+}$cells were increased $3 \mathrm{dpP}$ compared to controls. There was an approximately 3-fold increase in the proportion of lineage traced, Tom ${ }^{+} \mathrm{YARG}^{+}$cells $(0.7 \%)$, suggesting that CCR2+ monocytes can give rise to $\mathrm{M} 2$-like macrophages. However, the majority of $\mathrm{YARG}^{+}$cells was not lineage 
traced, presumably derived from resident macrophages, CCR2 ${ }^{\text {neg }}$ monocytes or escaped recombination (Figure 2G- $\mathrm{H}$ ).

We sought to localize cells derived from lineage traced CCR2+ monocytes during injury and repair of the tracheal epithelium. We delivered a single dose of tamoxifen to Ccr2CreER ${ }^{\mathrm{T} 2} ; \mathrm{ROSA}$-tdTom mice and injured the epithelium with polidocanol 4 days later. Tracheas were harvest for histology 1, 3 and $7 \mathrm{dpP}$ (Figure S2E). As expected, we observed $\mathrm{Tom}^{+}$cells in the submucosa beneath the tracheal epithelium in sham and injured tracheas at all times examined. To our surprise, we also observed an increased number of Tom ${ }^{+}$cells within the regenerating epithelial layer of the trachea at 3 and $7 \mathrm{dpP}$ (Figure 2I). This unexpected finding led us to confirm the existence of CD45+ leukocytes within the pseudostratified tracheal epithelium of sham and injured wild type mice (Figure 2J). Significantly, very few of these intraepithelial leukocytes are lineage traced in sham Ccr2-CreER ${ }^{T 2} ;$ ROSA-tdTom $^{+}$mice at any time (Figure 2I). Together these data show: (1) a dramatic increase in the number of $\mathrm{YARG}^{+} \mathrm{M} 2$-like macrophages after injury, (2) a population of myeloid cells within the tracheal epithelium that is not labelled in Ccr2CreER ${ }^{\mathrm{T} 2} ; \mathrm{ROSA}^{-t d T o m}{ }^{+}$mice under steady state conditions and (3) increases in the numbers of Ccr2-CreER ${ }^{\mathrm{T} 2} ; \mathrm{ROSA}^{-\mathrm{tdTOm}^{+}}$lineage traced cells in the tracheal mesenchyme and within the epithelium following injury.

\section{A population of myeloid cells is found within the pseudostratified tracheal epithelium}

Next, we focused on the population of CD45+ leukocytes within the tracheal epithelium. We peeled sheets of epithelium away from the underlying mesenchyme following a gentle enzymatic digestion as previously described (Rock et al., 2009). Immunofluorescence on isolated sheets revealed CD45+ (Figure S2F) and F4/80+ (Figure 2K) myeloid cells within the epithelial layer in close proximity to $\mathrm{KRT5}^{+}$basal cells. We similarly analyzed the tracheas of transgenic mice that express GFP under the control of the CSF1R locus, a broad marker of myeloid lineages (cFMS::eGFP, Figure S2I) (Sasmono et al., 2003). Immunofluorescence shows that the intraepithelial CD45+ and F4/80+ cells also express 
cFMS::eGFP+ (Figure S2J). We called these cells intraepithelial airway macrophages (IAMs).

\section{Most intraepithelial myeloid cells are molecularly distinct from other pulmonary myeloid lineages}

We used the 15-color myeloid panel (Figure 1, Supplemental Table S1) to compare IAMs to cells from the tracheal mesenchyme and lung parenchyma (containing alveoli and intralobar airways). We performed opt-SNE embedding of flow cytometry data from equal numbers of live, extravascular CD45+ cells from each of the 3 tissues (Figure $3 A$ ). Cell identities were assigned (Figure 3B) based on marker expression (Supplemental Table S1 and (Misharin et al., 2013)). Because the gating strategy was developed for dissociated lung parenchyma, the majority of cells from this tissue were contained within the conventional gates (Figure 3B). In contrast, the majority of myeloid cells from the tracheal epithelium fell outside of these gates, suggesting novel myeloid populations or cell states associate with the trachea under homeostasis (Figure S3A-B and light grey cells in Figure 3B). The few cells from the tracheal epithelium that fell within traditional gates were mostly macrophages and monocytes (Figure 3B) and a few DCs. The tracheal mesenchyme and lung parenchyma contained abundant CD103+ and CD11 $\mathrm{b}^{+} \mathrm{DCs}$. It is interesting to note that some cell types, such as macrophages and DCs, were observed in all 3 tissues, but exhibited slight differences in cell surface profiles represented as small shifts in tSNE space (Figure 3B). As expected, both the peeled tracheal epithelium and the underlying tracheal mesenchyme were devoid of SiglecF+ AMs (Figure 3B).

\section{IAMs, IMs and AMs are molecularly distinct from each other}

Because the conventional gating above pointed to unique myeloid populations in the tracheal epithelium and mesenchyme, we performed unbiased SCSeq of CD45+ leukocytes isolated from the tracheal epithelium, and CD45+;F4/80+ cells from the tracheal mesenchyme and lung parenchyma as described above (Figure 3C, S3C). Unsupervised clustering visualized in UMAP space showed that the majority of cells from each tissue formed distinct clusters (Figure 3D). Louvain clustering of these data with a 
resolution of 0.25 resulted in 12 populations (Figure 3E). Cells in cluster 4 were negative for Adgre 1 and expressed various lymphocyte markers. Cells in cluster 6 and 11 were negative for Ptprc (CD45) and Adgre1. Cells from Cluster 6 expressed markers associated with fibroblasts (Col3a1, Clec $3 b$ ) and cells in cluster 11 expressed markers of lung epithelium (Lamp3, Ager). Therefore clusters 4, 6 and 11 were characterized as nonmyeloid cells and excluded from further analysis (Figure S3D, crossed out). We characterized the remaining clusters by identifying the top 10 differentially expressed genes for each (Figure 3F-G, Figure S3E, Supplemental Table S3).

Clusters 0, 1 and 3 were from the tracheal mesenchyme and could be identified as classic macrophages (cluster 0), monocytes (cluster 1) and cells with an intermittent signature (cluster 3) (Figure 3E-G). These three populations are similar to previously identified IMs from the lung, due to their expression of Mertk, Fcgr1 and Cd68, combined with their negativity for SiglecF and Marco and variable expression of H2-ab1, H2-aa and Retnla (Gibbings et al., 2017; Ural et al., 2020). A similar signature was also observed for Cluster 8 that originated from the lung, presumably representing the captured lung IMs.

Clusters 5, 7 and 9 were isolated from the tracheal epithelium. Contrary to previous reports (Sertl et al., 1986), these cells do not resemble DCs. Of particular interest was Cluster 7 that contained IAMs. These macrophages are enriched for the expression of Fcgr1, Cd68 and Adgre1 (Figure 2G) but also express unique markers such as Tgfßr1 and Scimp (Cluster 7, Supplemental Table S3). Importantly, the transcription profile of these cells was distinct from those reported for IMs (Gibbings et al., 2017), and could not be binned into a classical category. IAMs were also negative for the marker Cd169, recently associated with a subpopulation of macrophages localized in close proximity to airway neurons (Ural et al., 2020). Cluster 5 expressed genes associated with neutrophils, and cluster 9 expressed markers of lymphocytes.

Consistent with the flow cytometry data presented above, the cells isolated from the lung parenchyma expressed genes associated with AMs (Cluster 2) and a smaller population 
of IMs (Cluster 8). Cells in Cluster 10, also isolated from the lung, expressed markers of lymphocytes (Figure 3G, Supplemental Table S3).

For technical reasons, we were unable to separate the epithelium from the underlying mesenchyme after injury prior to SCSeq (Figure 2A-E). Therefore, we derived a transcriptional signature from the top 30 expressed genes in the IAM cluster (Cluster 7 Fig 3E-G, Supplemental Table S4) and analyzed the expression of this signature in the post-injury SCSeq data described above (Figure 2A-E). Cells enriched for this gene signature fell into the monocyte/macrophage cluster (Cluster 3). Consistent with our immunofluorescence data, the IAM gene signature was lowest at $1 \mathrm{dpP}$, when most epithelial cells have died and been sloughed (Figure 3J, Figure S3F). The accumulation of CCR2 lineage traced cells (Figure 2I) concomitant with an increase in the number of cells with an IAM transcriptional signature (Figure 3J and Figure S3F) supports a model in which $\mathrm{CCR}^{+}$monocytes replace IAMs that are lost as a result of epithelial injury.

\section{Type 2 cytokines modulate basal cell behaviors in vitro}

Next, we turned our attention to type 2 cytokines, required for the polarization of M2-like macrophages (Wynn et al., 2013) that increase in the trachea after injury (Figure 2A-C). Our SCSeq of CD45+ cells during tracheal injury and repair identified mast cells and group 2 innate lymphoid cells as sources of //13 after injury (data not shown). We sought to determine whether these cytokines also directly affect basal cells. We cultured basal cells in the tracheosphere organoid assay (Rock et al., 2009) and supplemented the medium with cytokines associated with lung injury including IL1 $\beta$, IL2, IL4, IL5 and IL13 (Lechner et al., 2017; Tadokoro et al., 2014) (Figure S4A-B). As previously described (Danahay et al., 2015) the addition of either IL4 or IL13 resulted in an increase in average sphere size (Figure S4A). IL4 and IL13 both signal via a common receptor subunit, IL4R $\alpha$. We analyzed a previously published dataset (Plasschaert et al., 2018) and found that //4ra is expressed on some basal cells. Indeed, flow cytometry on isolated basal cells demonstrated that $\sim 11 \%$ of basal cells express IL4R $\alpha$ (Figure $4 \mathrm{~A}$ ). Based on this finding, we tested whether IL4 and IL13 signal directly to basal cells. We infected 2D adherent 
cultures of IL4R $\alpha$ flox/flox basal cells with an adenovirus expressing Cre-GFP fusion protein to delete the floxed //4r $\alpha$ receptor subunit. Three days after infection, Ad5CMVCre-eGFP infected cells, but not AdCMV-GFP controls, had lost IL4R $\alpha$ expression (Figure S4C). When grown as organoids in the presence or absence of IL4 or IL13 the IL4R $\alpha$-deficient cells did not respond to the cytokines, unlike control cells (Figure 4C- D).

\section{Myeloid cells promote repair of the pseudostratified epithelium}

To assess the role of immune cells on tracheal repair in vivo, we injured CIAE-NOG animals that lack an adaptive immune system and have a dysfunctional innate immune system including lack of monocytes and macrophages. Three days after polidocanolinduced epithelial injury, basal cells had not undergone extensive self-renewal and temporary stratification, a normal step in the repair process (compare Figure S4D-E to Figure S1D). These data suggest a crucial role of the immune system in fostering epithelial repair. Since there is an accumulation of CCR2 lineage traced cells within the regenerating tracheal epithelium (Figure $2 \mathrm{I}$ ), we reasoned that CCR2+ monocytes and their progeny are required for epithelial repair. To test this hypothesis, we analyzed epithelial repair in mice homozygous for a Ccr2-RFP allele that lack Ccr2 expression and do not have functioning CCR2+ myeloid cells (Saederup et al., 2010) (Figure S4F). Importantly, there were no developmental abnormalities of the tracheal epithelium of Ccr2-RFP homozygotes. Histology and immunofluorescence demonstrated incomplete epithelial repair in Ccr2-deficient mice compared to heterozygous littermate controls (Figure 4E-F), characterized by a lack of multiciliated cells.

\section{Discussion}

The mouse trachea is a well characterised model to study airway biology which shares many morphological and histological features with small human airways (Rock et al., 2010). In this study, we used the trachea to interrogate the myeloid cells and their role in homeostasis and epithelial injury and repair. Under steady state conditions we identified macrophages in the tracheal submucosa that are similar to recently described interstitial macrophages (IMs), providing further rationale for our use of the trachea as an airway 
model. We unexpectedly identified a population of macrophages within the tracheal epithelium that are molecularly distinct from IMs and AMs. These intraepithelial macrophages (IAMs) are also distinct from DCs which have been described within the epithelium (Sertl et al., 1986). Similarly, Langerhans cells, previously considered a specialized dendritic cell of the epidermis, have recently been reclassified as a tissue resident macrophage population (Doebel et al., 2017). Under baseline conditions, both IAMs and Langerhans cells are quiescent, defined by negativity for cell cycle markers (Ccne2, Aurka, Mki67) in SCSeq, absence of Ki67 by immunostaining (data not shown), and directly contact surrounding epithelial cells (Clayton et al., 2017; Doebel et al., 2017).

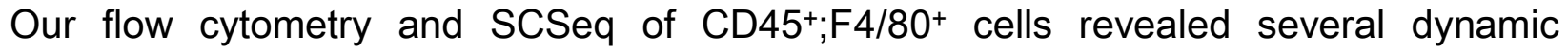
properties of trachea-associated myeloid populations in response to epithelial injury. For example, at 1 and $3 \mathrm{dpP}$ we detected an increase in neutrophils, monocytes and macrophages. Neutrophils are thought to be key in modulating the cytokine environment to foster recruitment and differentiation of other immune players (Blázquez-Prieto et al., 2018; Kaplanski et al., 2003; Lechner et al., 2017; Mindt et al., 2018; Ricardo-Gonzalez et al., 2018). In the monocyte and macrophage compartment, we observed a shift toward M2-like polarization of macrophages. We confirmed the accumulation of Arginaseexpressing M2-like macrophages after epithelial injury using transgenic reporter mice.

By combining the Arginase reporter allele with a Ccr2-based lineage tracing strategy, we provide evidence that some of the M2-like macrophages come from a Ccr2-expressing precursor. Similar to previous reports, we also observed Cx3cr1-expressing monocytes in homeostatic tracheas and in response to injury (Joshi et al., 2020; Ural et al., 2020). Whether or not these cells also give rise to M2-like macrophages and IAMs remains unknown. The accumulation of CCR2-derived cells concomitant with the reemergence of the IAM transcriptional signature 4 and $7 \mathrm{dpP}$ suggests that CCR2 ${ }^{+}$monocytes are a precursor for IAMs in response to injury.

Monocyte-derived, alternatively activated, M2-like macrophages are crurcial for development, regeneration and remodeling in disease (Byrne et al., 2015; Lechner et al., 
2017). Our previous work demonstrated the importance of M2-like macrophages for the compensatory regrowth of alveoli in response to pneumonectomy in mice (Lechner et al., 2017). We used parallel strategies to determine whether leukocytes also promote the repair of the tracheal epithelium. First, we performed polidocanol-injury of the airway epithelium in immunodeficient mice and demonstrated a deficiency in basal cell proliferation and differentiation 3 days post-injury. Second, we analyzed tracheal repair in Ccr2-deficient mice to more specifically determine whether monocytes and their progeny are required for epithelial repair. Similar to immunodeficient mice, there was inefficient repair of the tracheal epithelium in Ccr2-deficient mice, characterized by failure to generate a pseudostratified epithelium with multiciliated cells beginning 3 days postinjury.

Our SCSeq data of $\mathrm{CD} 45^{+} ; \mathrm{F} 4 / 80^{+}$cells revealed that the type 2 cytokines, IL4 and IL13, are expressed by group 2 innate lymphoid cells and mast cells in the trachea (data not shown). In addition to numerous other functions, these factors are critical for the polarization of M2-like macrophages. Future experiments will identify the relative contributions of type 2 cytokines by mast cells, ILC2s and other non-myeloid lineages. Because we detected IL4 and IL13 in the injured trachea, we sought to confirm previous reports that these cytokines directly modulate epithelial basal cell behaviors (Danahay et al., 2015; Kuperman et al., 2002). The treatment of wild type basal cells (but not //4rodeficient basal cells) with IL4 or IL13 resulted in an increased size of tracheospheres. The cellular basis for this increase remains to be determined, but highlights the pleotropic effects of type 2 cytokines during repair of the tracheal epithelium.

This work and recent work by others (Chakarov et al., 2019; Gibbings et al., 2017; Lechner et al., 2017; Misharin et al., 2013; Ural et al., 2020) illustrate the striking heterogeneity within the myeloid compartment of the respiratory system. With modern, single cell technologies we can slowly appreciate the high complexity of tissue resident monocytes and macrophages which will be key for the development of future, targeted technologies and therapies (Klichinsky et al., 2020). 


\section{Contact for Reagents}

This study did not generate new, unique reagents. Datasets are available in repositories. Further information and requests for resources and reagents should be directed to, and will be fulfilled by, the Lead Contact, Jason R. Rock (rockjr@bu.edu).

\section{Author Contributions:}

Conceptualization: AEE, SAM, JRR; Methodology and Investigation AEE, ABY, RMFP, ACB, HH, NM, and HR; Data Analysis and Interpretation: AEE, CVM, ACB and JRR; Writing, AEE and JRR, Writing - Review and Editing; AEE, ABY, ACB, SAM and JRR; Supervision, Project Administration, and Funding Acquisition, SAM and JRR.

Funding: This research was funded by the National Institutes of Health grant numbers R01 HL127002 U01 HL148692 and U01 HL134766 (JRR); and by a sponsored research agreement from Janssen Pharmaceuticals (SAM).

\section{Acknowledgments:}

We would like to thank the entire Rock lab for helpful discussions. We thank Drs. Darrell Kotton, Brigid Hogan and Jay Mizgerd for helpful comments on the manuscript. We thank Brian Tilton of the Boston University School of Medicine Flow Cytometry core Facility, Dr. Yuriy Alekseyev and Ashley LeClerc of the Boston University School of Medicine Sequencing core and Dr Michael T.

Kirber Boston University School of Medicine Cellular imaging core for excellent technical assistance.

\section{Conflicts of Interest:}

SAM received sponsored research funding from Janssen Pharmaceuticals. 
bioRxiv preprint doi: https://doi.org/10.1101/2020 06 16.146035; this version posted June 16, 2020. The copyright holder for this preprint (which was not certified by peer review) is the author/funder, who has granted bioRxiv a license to display the preprint in perpetuity. It is made available under aCC-BY-NC-ND 4.0 International license.

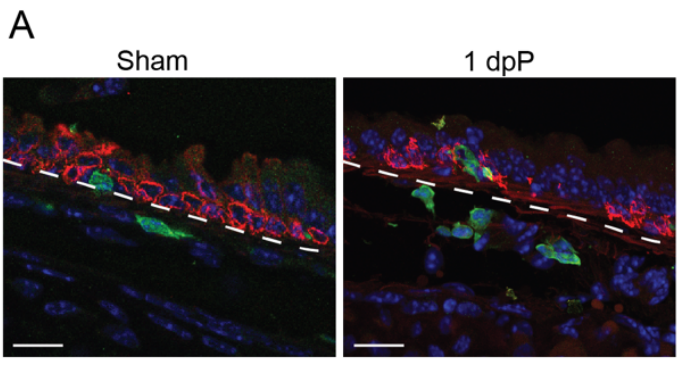

Krt5 CD45 Hoechst
B

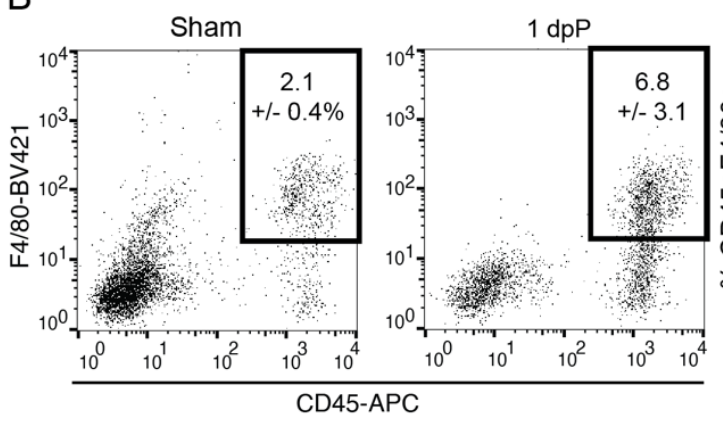

口Sham

- Polidocanol

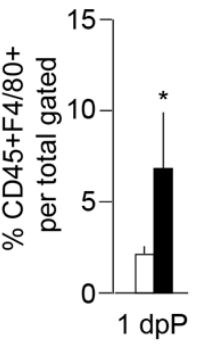

C

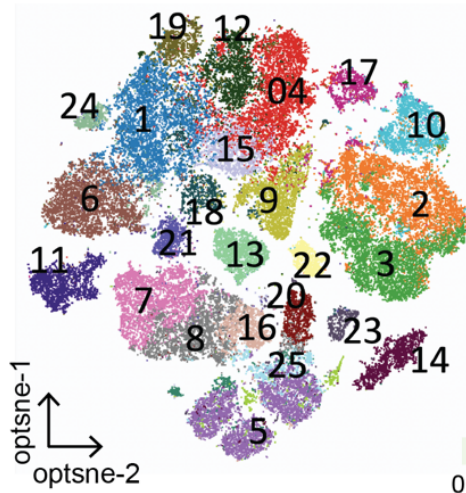

D

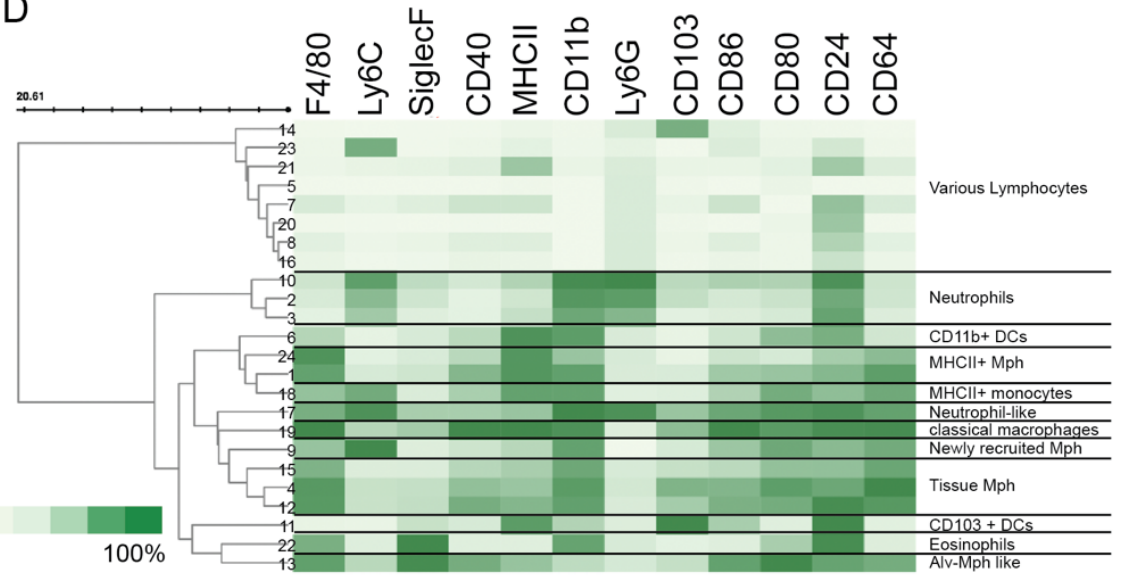

E

Sham

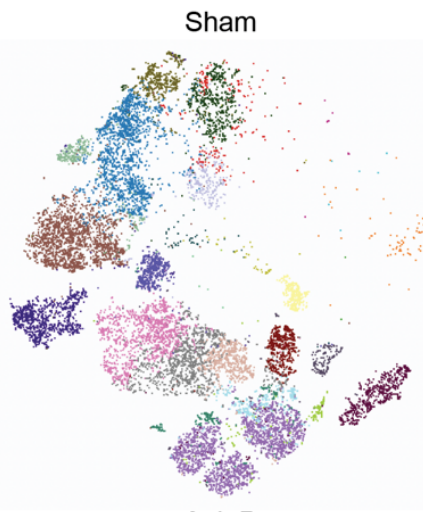

$3 \mathrm{dpP}$

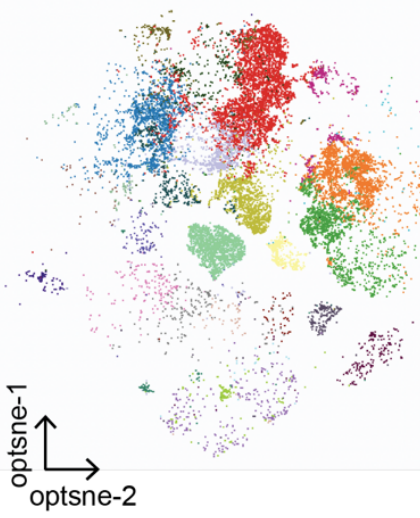

F

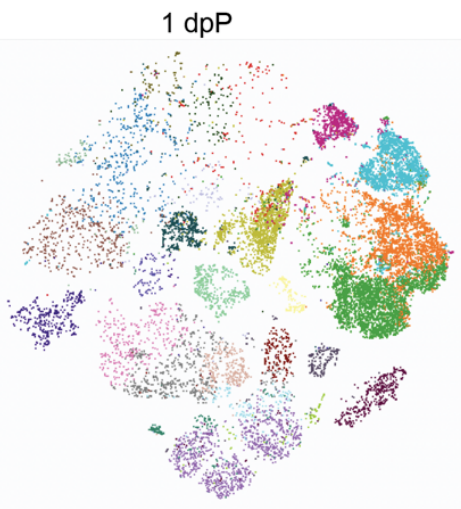

$7 \mathrm{dpP}$

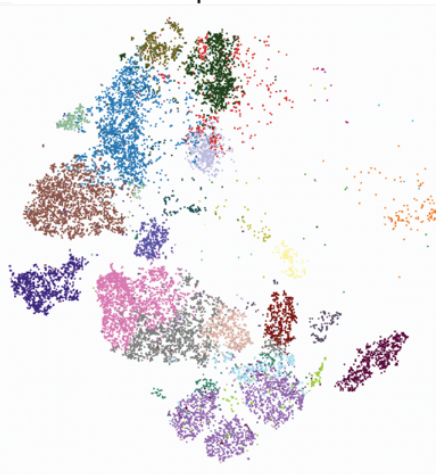

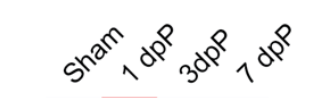

10
2 Neutrophils

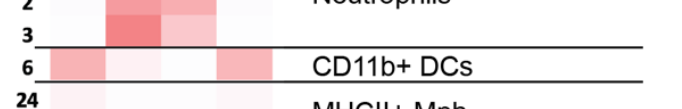

\begin{tabular}{c|c|}
24 & $\mathrm{MHCll}+\mathrm{Mph}$ \\
1 &
\end{tabular}

\begin{tabular}{cl}
\hline 18 & MHCll+ monocytes \\
\hline 17 & Neutrophil-like \\
\hline $\mathbf{1 5}$ & Classical macrophages \\
\hline $\mathbf{1 5}$ & Newly recruited Mph \\
\hline $\mathbf{1 2}$ & Tissue Mph \\
\hline $\mathbf{1 2}$ & \\
\hline 13 & CD103 + DCs \\
\hline
\end{tabular}

14

23

21
5
7

7
20

8
16

16

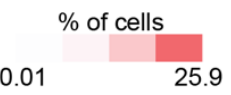

Various Lymphocytes 
Figure 1: Temporal changes of the tracheal myeloid compartment following injury; (A) Immunofluorescence on sections of trachea show an increase in the number of CD45+ cells (green) near basal cells (KRT5, red) following polidocanol-induced injury. Scale bar $25 \mu \mathrm{m}$ (B) FACS analysis of CD45+F4/80+ myeloid cells after $1 \mathrm{dpP}$; Percentage of CD45+F4/80+ cells of total $(n=4) ;(C)$ Unsupervised clustering of all cells combined from sham, 1-, 3-, and 7dpP based on 15-marker myeloid panel; ( $n=1 /$ timepoint, consistent of 2 animals) (D) Heatmap of marker expression data upon which cluster annotations were based, scaled from 0 to $100 \%$ of normalized, arcsin value of fluorescent intensity (E) Cells isolated on indicated days post-injury, visualized by cluster assignment; $(F)$ Relative abundance of cells from 24 distinct clusters across injury and repair, scaled 0.01 to $25.9 \%$ of total cells in analysis. 
bioRxiv preprint doi: https://doi.org/10.1101/2020.06.16.146035; this version posted June 16, 2020. The copyright holder for this preprint (which was not certified by peer review) is the author/funder, who has granted bioRxiv a license to display the preprint in perpetuity. It is made available under aCC-BY-NC-ND 4.0 International license.
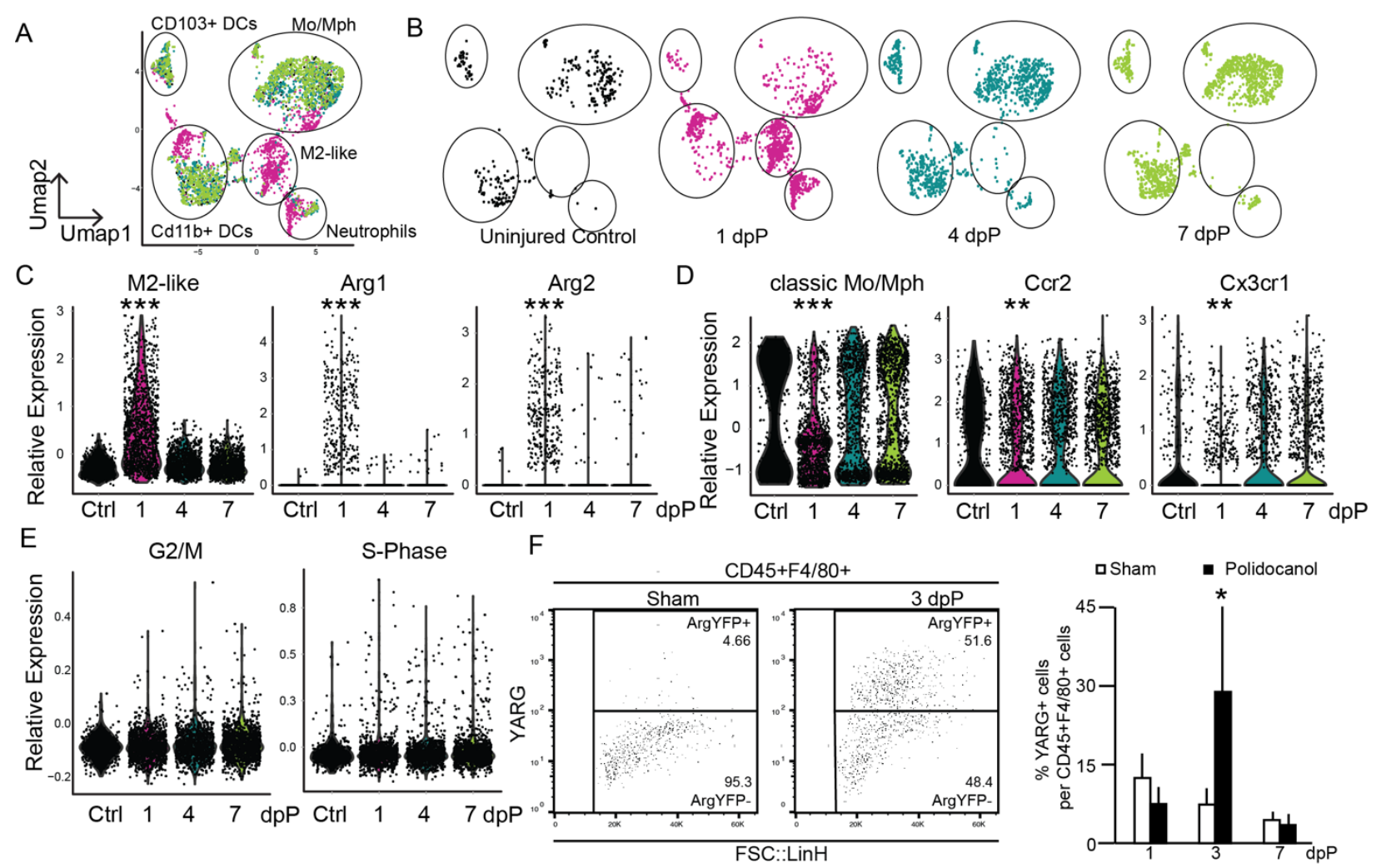

G

CD45+
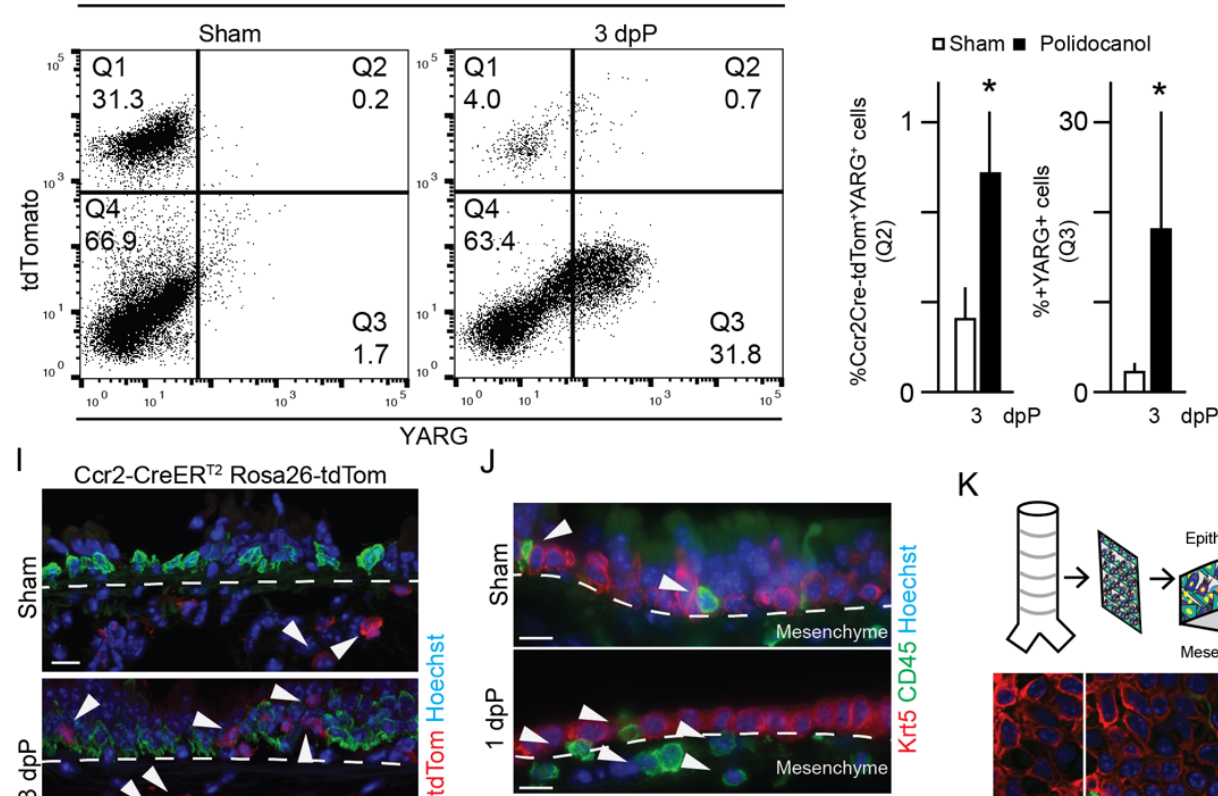

$\mathrm{K}$
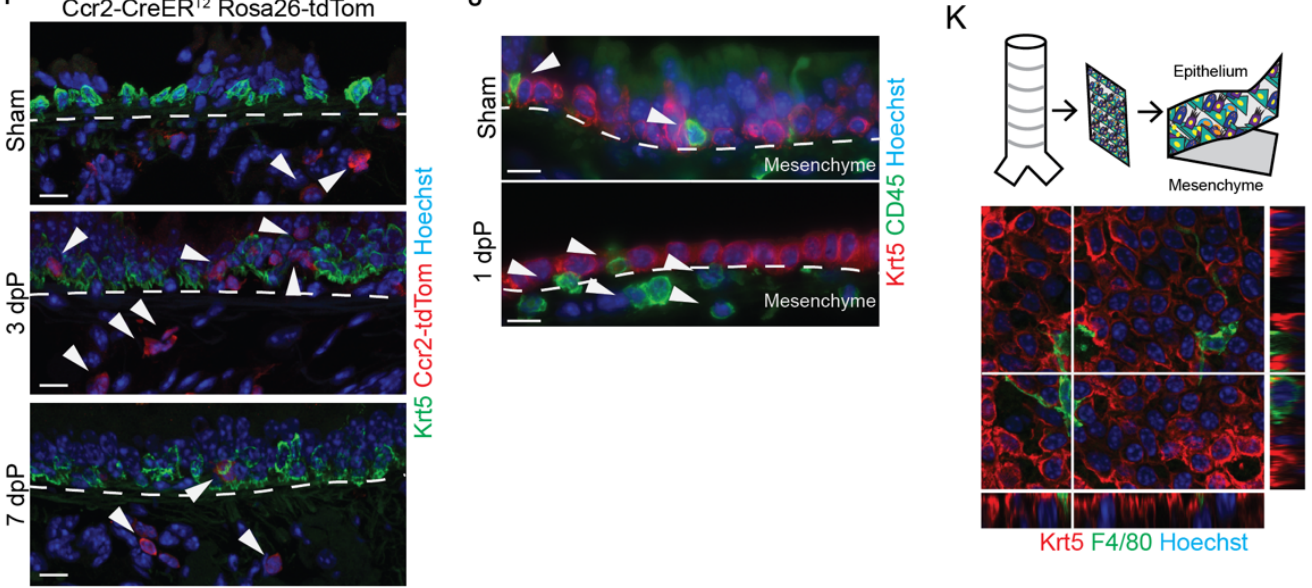
Figure 2: Unbiased analysis of tracheal myeloid populations after injury; (A) UMAP visualization of all SCSeq data across airway injury and repair overlaid; ( $n=1 /$ time point, consistent of 3 animals); captured cells: uninjured $=458,1 \mathrm{dpP}=1625,4 \mathrm{dpP}=1507,7 \mathrm{dpP}=1717$ (B) UMAP visualization of SCSeq data from individual time points. From left to right, uninjured controls, 1dpP, 4dpP and 7dpP; (C) Violin plots M2-like macrophage gene signature and Arg1 and Arg2 (D), Violin plots classic monocyte/macrophage gene signature and Ccr2 and Cx3cr1; (E) FACS Analysis of dissociated tracheas from $\mathrm{YARG}^{+}$mice gated on $\mathrm{CD}^{+*} ; \mathrm{F} 4 / 80^{+}$( $\mathrm{n}(\mathrm{Sham})=3$, $\mathrm{n}(3 \mathrm{dpP})=3)$; (F) Quantification of YARG FACS data (G) FACS Analysis of dissociated tracheas from Ccr2-CreERT2;ROSA-tdTom, YARG animals after tamoxifen administration and Sham or Polidocanol treatment $(n(S h a m)=4, n(3 d p P)=3) ;(H)$ Quantification of FACS results; (I) Immunofluorescence analysis of Ccr2-CreERT2;ROSA-tdTom animals during injury and repair; Krt5 (green), Ccr2-CreER ${ }^{\top 2}$;ROSA-tdTom lineage trace (red), and nuclear stain Höechst (blue). scale bar $15 \mu \mathrm{m}$; (J) Histology of wild type animals; Krt5 (red), CD45 (green), nuclear stain Höechst, scale bar $15 \mu \mathrm{m}$; (K) Immunofluorescence on peeled epithelial sheet showing F4/80+ myeloid cells (green) Krt5+ epithelial basal cells (red). nuclear stain Höechst (blue); 
bioRxiv preprint doi: https://doi.org/10.1101/2020 06 16.146035; this version posted June 16, 2020. The copyright holder for this preprint (which was not certified by peer review) is the author/funder, who has granted bioRxiv a license to display the preprint in perpetuity. It is made available under aCC-BY-NC-ND 4.0 International license.
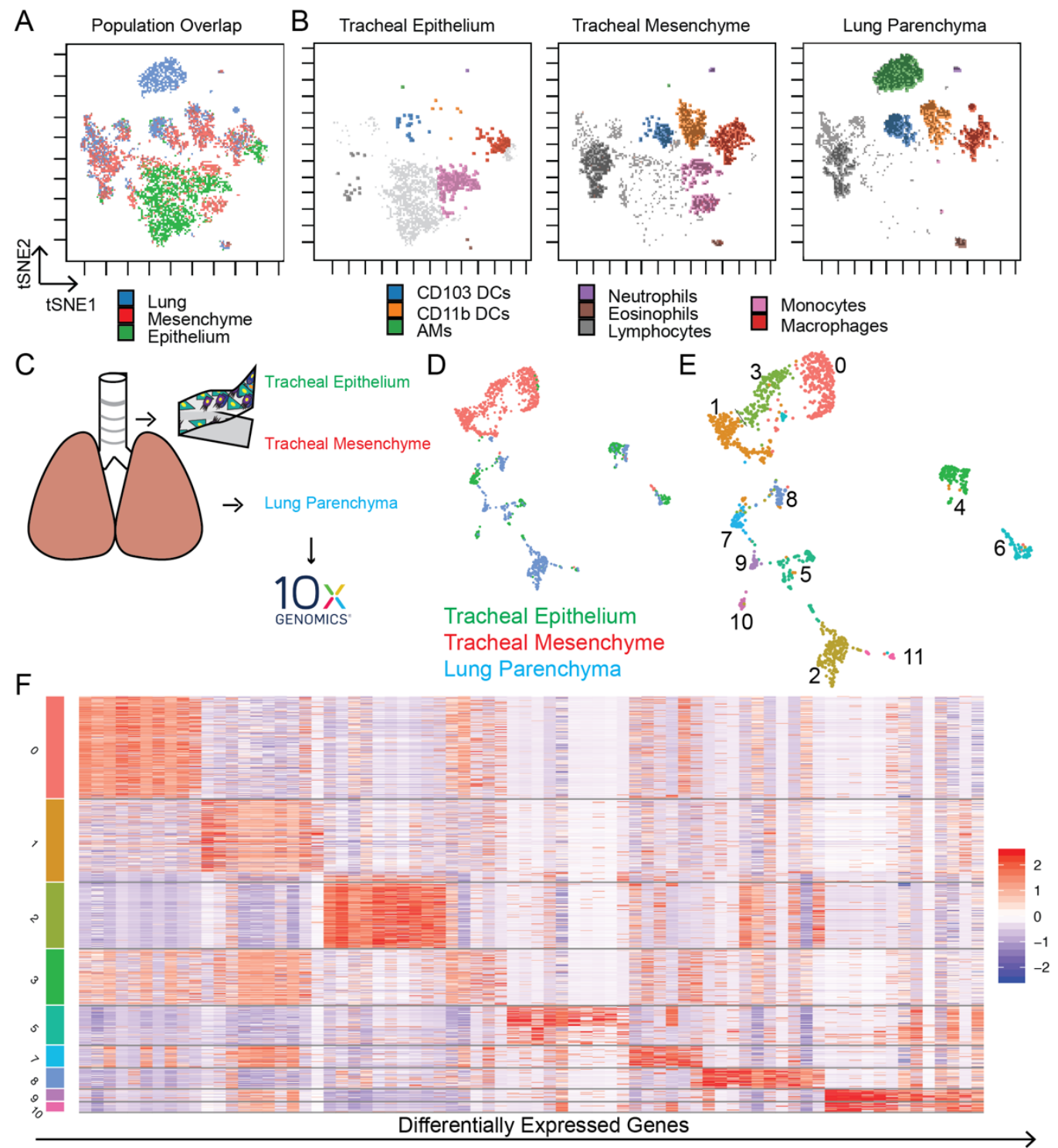

G

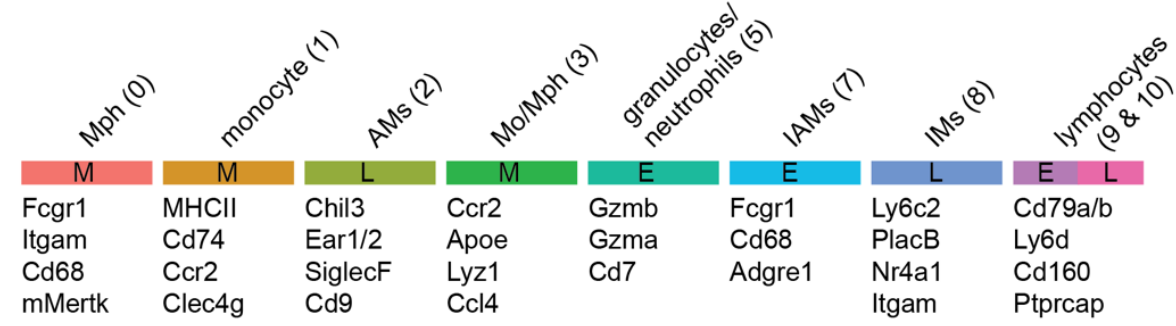

$\mathrm{H} \quad$ Projection of IAM signature onto injury dataset (Figure 2)

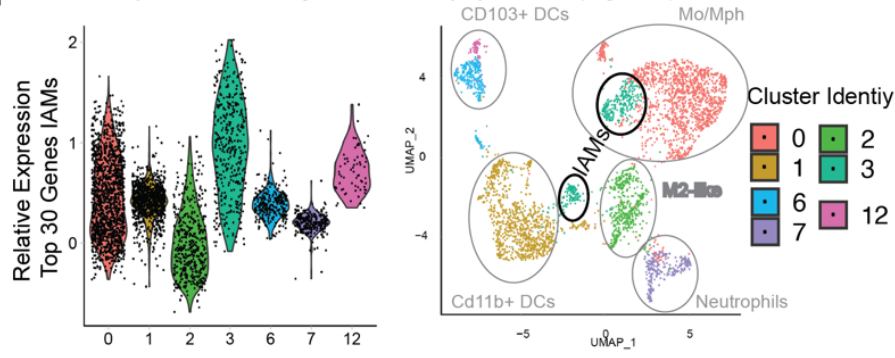

J IAM signature over injury timecourse

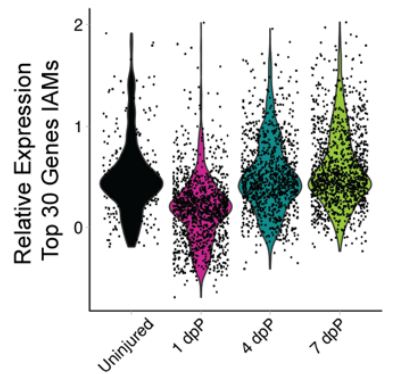


Figure 3: Myeloid cells isolated from the epithelium, mesenchyme and lung parenchyma have distinct transcriptional and translational identities; (A) Unsupervised clustering of 15-marker myeloid panel, FACS analysis of cells from lung, mesenchyme and epithelium ( $n=5 /$ tissue pooled); (B) Unsupervised clustering of cells isolated from epithelium (left), mesenchyme (middle) and lung (right); (C) Schematic representation of samples isolated for SCSeq, ( $n=5 /$ tissue animals pooled); captured epithelium=329 cells, mesenchyme= 721 cells, lung= 460 cells; (D) Unsupervised clustering, UMAP, colored for tissue of origin; (E) Unsupervised Louvaine clustering at resolution 0.25 of SCSeq data; $(F)$ Heatmap of differentially expressed genes in myeloid clusters; Clusters 4, 6, and 11 excluded as contaminants for further details see Supplemental Table S3 (G) Cluster assignment based on selected transcripts and annotation of tissue of origin (E - epithelium, M - mesenchyme, L- lung) $(H)$ Projection of IAM gene signature (Cluster 7, Figure 3F) onto single cell injury dataset (Figure 2A-E), (J) Projection of IAM gene signature onto injury time points. 
bioRxiv preprint doi: https://doi.org/10.1101/2020.06.16.146035; this version posted June 16, 2020. The copyright holder for this preprint (which was not certified by peer review) is the author/funder, who has granted bioRxiv a license to display the preprint in perpetuity. It is made available under aCC-BY-NC-ND 4.0 International license.

A

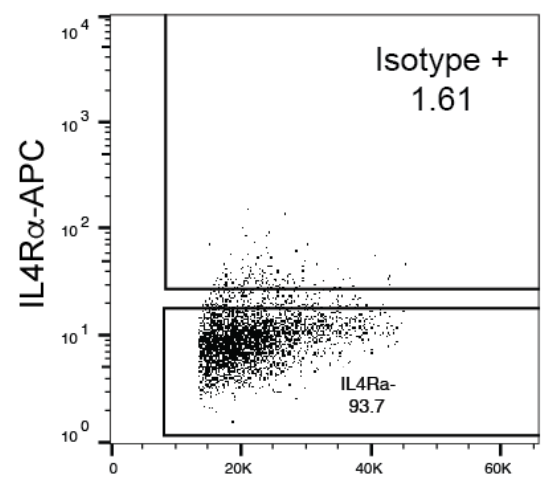

Live, Single Cells

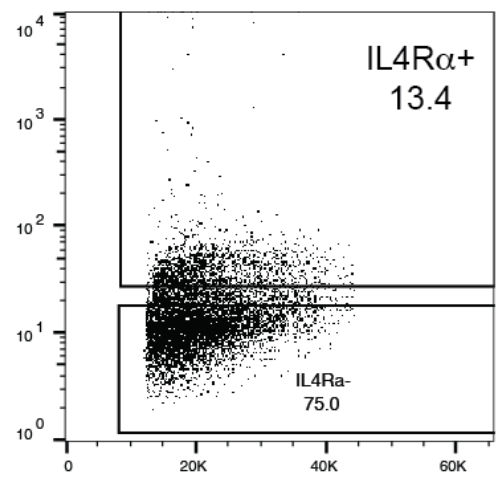

FSC-LinH

C
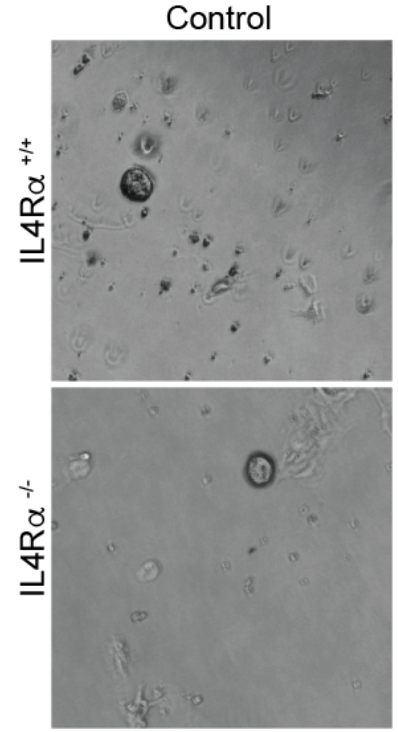

E
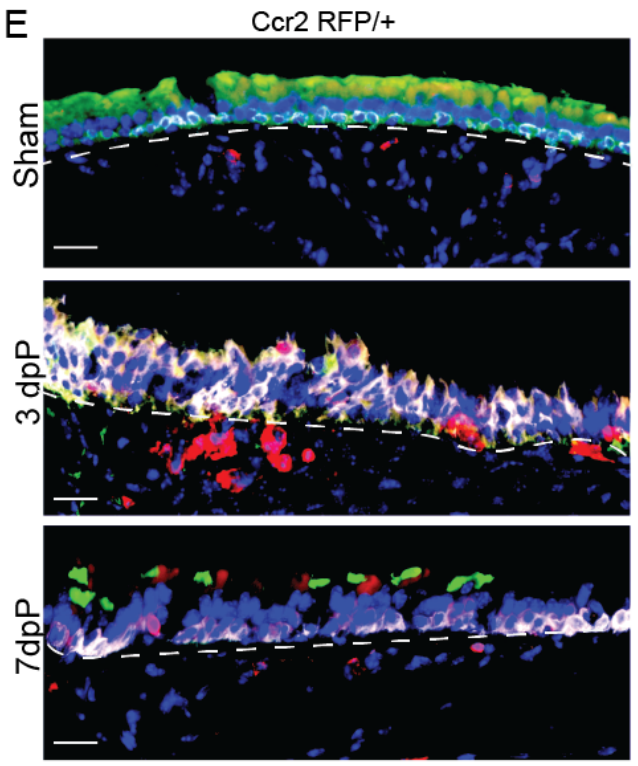

Krt5 Ccr2-RFP acetylated Tubulin Hoechst
B

+CMV-GFP or +CMV-Cre-GFP 3 day infection

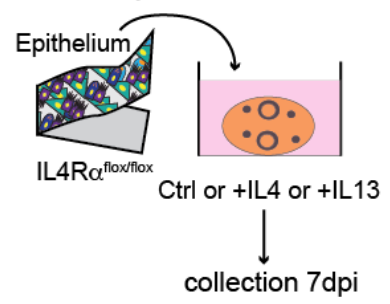

D

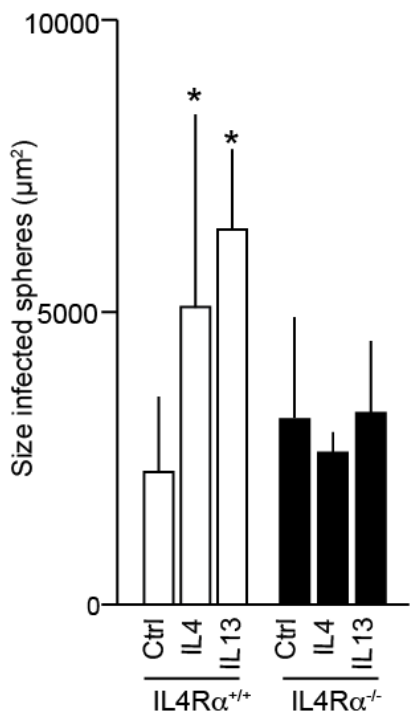

$\mathrm{F}$

口Sham घ Polidocanol

口Ccr2 RFP/+ $=\mathrm{Cc} 2 \mathrm{RFP} / \mathrm{RFP}$

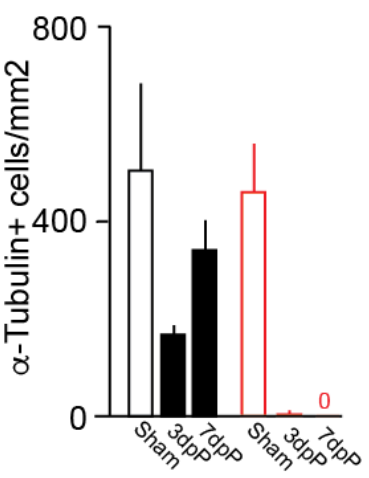


Figure 4: CCR2+ myeloid cells promote basal cell mediated epithelial repair (A) FACS analysis of IL4R $\alpha$ expression on basal cells $(n=3)$; (B) Schematic representation of basal cell isolation and infection for conditional deletion of IL4R $\alpha$ and addition of IL4 or IL13 in tracheosphere culture (C) Culture of IL4R $\alpha^{+/+}(n=3)$ and IL4R $\alpha^{-/-}(n=3)$ basal cells in the presence of IL4 or IL13; (D) Quantification of sphere size after 10 days of culture; (E) Epithelial repair in CCR2-deficient mice and heterozygous controls $3 \mathrm{dpP}$ and $7 \mathrm{dpP}$ (heterozygote $\mathrm{n}($ Sham) $=2$, $\mathrm{n}$ (injury) $=3$ each; homozygote $n($ Sham $)=4$; $n$ (injury)=3 each), scale bars $25 \mu \mathrm{m}$ and (F) Quantification of acetylated $\alpha$-Tubulin ${ }^{+}$ciliated cells as a metric of repair. 


\section{References}

Anderson, K.G., Mayer-Barber, K., Sung, H., Beura, L., James, B.R., Taylor, J.J., Qunaj, L., Griffith, T.S., Vezys, V., Barber, D.L., et al. (2014). Intravascular staining for discrimination of vascular and tissue leukocytes. Nat. Protoc.

Belkina, A.C., Ciccolella, C.O., Anno, R., Halpert, R., Spidlen, J., and Snyder-Cappione, J.E. (2019). Automated optimized parameters for T-distributed stochastic neighbor embedding improve visualization and analysis of large datasets. Nat. Commun.

Blázquez-Prieto, J., López-Alonso, I., Huidobro, C., and Albaiceta, G.M. (2018). The emerging role of neutrophils in repair after acute lung injury. Am. J. Respir. Cell Mol. Biol.

Borthwick, D.W., Shahbazian, M., Krantz, Q.T., Dorin, J.R., and Randell, S.H. (2001). Evidence for stem-cell niches in the tracheal epithelium. Am. J. Respir. Cell Mol. Biol.

Byrne, A.J., Mathie, S.A., Gregory, L.G., and Lloyd, C.M. (2015). Pulmonary macrophages: Key players in the innate defence of the airways. Thorax.

Chakarov, S., Lim, H.Y., Tan, L., Lim, S.Y., See, P., Lum, J., Zhang, X.-M., Foo, S., Nakamizo, S., Duan, K., et al. (2019). Two distinct. Science (80-. ).

Clayton, K., Vallejo, A.F., Davies, J., Sirvent, S., and Polak, M.E. (2017). Langerhans cellsprogrammed by the epidermis. Front. Immunol.

Danahay, H., Pessotti, A.D., Coote, J., Montgomery, B.E., Xia, D., Wilson, A., Yang, H., Wang, Z., Bevan, L., Thomas, C., et al. (2015). Notch2 is required for inflammatory cytokine-driven goblet cell metaplasia in the lung. Cell Rep.

Doebel, T., Voisin, B., and Nagao, K. (2017). Langerhans Cells - The Macrophage in Dendritic Cell Clothing. Trends Immunol.

Finak, G., McDavid, A., Yajima, M., Deng, J., Gersuk, V., Shalek, A.K., Slichter, C.K., Miller, H.W., McElrath, M.J., Prlic, M., et al. (2015). MAST: A flexible statistical framework for assessing transcriptional changes and characterizing heterogeneity in single-cell RNA sequencing data. Genome Biol.

Gibbings, S.L., Thomas, S.M., Atif, S.M., McCubbrey, A.L., Desch, A.N., Danhorn, T., Leach, S.M., Bratton, D.L., Henson, P.M., Janssen, W.J., et al. (2017). Three unique interstitial macrophages in the murine lung at steady state. Am. J. Respir. Cell Mol. Biol.

Joshi, N., Watanabe, S., Verma, R., Jablonski, R.P., Chen, C.I., Cheresh, P., Markov, N.S., 
Reyfman, P.A., McQuattie-Pimentel, A.C., Sichizya, L., et al. (2020). A spatially restricted fibrotic niche in pulmonary fibrosis is sustained by M-CSF/M-CSFR signalling in monocytederived alveolar macrophages. Eur. Respir. J.

Kaplanski, G., Marin, V., Montero-Julian, F., Mantovani, A., and Farnarier, C. (2003). IL-6: A regulator of the transition from neutrophil to monocyte recruitment during inflammation. Trends Immunol.

Kawano, H., Kayama, H., Nakama, T., Hashimoto, T., Umemoto, E., and Takeda, K. (2016). IL10-producing lung interstitial macrophages prevent neutrophilic asthma. Int. Immunol.

Klichinsky, M., Ruella, M., Shestova, O., Lu, X.M., Best, A., Zeeman, M., Schmierer, M., Gabrusiewicz, K., Anderson, N.R., Petty, N.E., et al. (2020). Human chimeric antigen receptor macrophages for cancer immunotherapy. Nat. Biotechnol.

Kuperman, D.A., Huang, X., Koth, L.L., Chang, G.H., Dolganov, G.M., Zhu, Z., Elias, J.A., Sheppard, D., and Erle, D.J. (2002). Direct effects of interleukin-13 on epithelial cells cause airway hyperreactivity and mucus overproduction in asthma. Nat. Med.

Lechner, A.J., Driver, I.H., Lee, J., Conroy, C.M., Nagle, A., Locksley, R.M., and Rock, J.R. (2017). Recruited Monocytes and Type 2 Immunity Promote Lung Regeneration following Pneumonectomy. Cell Stem Cell.

Levine, J.H., Simonds, E.F., Bendall, S.C., Davis, K.L., Amir, E.A.D., Tadmor, M.D., Litvin, O., Fienberg, H.G., Jager, A., Zunder, E.R., et al. (2015). Data-Driven Phenotypic Dissection of AML Reveals Progenitor-like Cells that Correlate with Prognosis. Cell.

Mindt, B.C., Fritz, J.H., and Duerr, C.U. (2018). Group 2 innate lymphoid cells in pulmonary immunity and tissue homeostasis. Front. Immunol.

Misharin, A. V., Morales-Nebreda, L., Mutlu, G.M., Budinger, G.R.S., and Perlman, H. (2013). Flow cytometric analysis of macrophages and dendritic cell subsets in the mouse lung. Am. J. Respir. Cell Mol. Biol.

Plasschaert, L.W., Žilionis, R., Choo-Wing, R., Savova, V., Knehr, J., Roma, G., Klein, A.M., and Jaffe, A.B. (2018). A single-cell atlas of the airway epithelium reveals the CFTR-rich pulmonary ionocyte. Nature.

Reese, T.A., Liang, H.E., Tager, A.M., Luster, A.D., Van Rooijen, N., Voehringer, D., and Locksley, R.M. (2007). Chitin induces accumulation in tissue of innate immune cells associated with allergy. Nature. 
Ricardo-Gonzalez, R.R., Van Dyken, S.J., Schneider, C., Lee, J., Nussbaum, J.C., Liang, H.E., Vaka, D., Eckalbar, W.L., Molofsky, A.B., Erle, D.J., et al. (2018). Tissue signals imprint ILC2 identity with anticipatory function. Nat. Immunol.

Rock, J.R., and Hogan, B.L.M. (2011). Epithelial Progenitor Cells in Lung Development, Maintenance, Repair, and Disease. Annu. Rev. Cell Dev. Biol.

Rock, J.R., Onaitis, M.W., Rawlins, E.L., Lu, Y., Clark, C.P., Xue, Y., Randell, S.H., and Hogan, B.L.M. (2009). Basal cells as stem cells of the mouse trachea and human airway epithelium. Proc. Natl. Acad. Sci.

Rock, J.R., Randell, S.H., and Hogan, B.L.M. (2010). Airway basal stem cells: a perspective on their roles in epithelial homeostasis and remodeling. Dis. Model. Mech.

Saederup, N., Cardona, A.E., Croft, K., Mizutani, M., Cotleur, A.C., Tsou, C.L., Ransohoff, R.M., and Charo, I.F. (2010). Selective chemokine receptor usage by central nervous system myeloid cells in CCR2-red fluorescent protein knock-in mice. PLoS One.

Sasmono, R.T., Oceandy, D., Pollard, J.W., Tong, W., Pavli, P., Wainwright, B.J., Ostrowski, M.C., Himes, S.R., and Hume, D.A. (2003). A macrophage colony-stimulating factor receptorgreen fluorescent protein transgene is expressed throughout the mononuclear phagocyte system of the mouse. Blood.

Sertl, K., Takemura, T., Tschachler, E., Ferrans, V.J., Kaliner, M.A., and Shevach, E.M. (1986). Dendritic cells with antigen-presenting capability reside in airway epithelium, lung parenchyma, and visceral pleura. J. Exp. Med.

Tadokoro, T., Wang, Y., Barak, L.S., Bai, Y., Randell, S.H., and Hogan, B.L.M. (2014). IL6/STAT3 promotes regeneration of airway ciliated cells from basal stem cells. Proc. Natl. Acad. Sci. U. S. A.

Tan, S.Y.S., and Krasnow, M.A. (2016). Developmental origin of lung macrophage diversity. Dev.

Ural, B.B., Yeung, S.T., Damani-Yokota, P., Devlin, J.C., de Vries, M., Vera-Licona, P., Samji, T., Sawai, C.M., Jang, G., Perez, O.A., et al. (2020). Identification of a nerve-associated, lungresident interstitial macrophage subset with distinct localization and immunoregulatory properties. Sci. Immunol.

Wynn, T.A., Chawla, A., and Pollard, J.W. (2013). Macrophage biology in development, homeostasis and disease. Nature. 
bioRxiv preprint doi: https://doi.org/10.1101/2020.06.16.146035; this version posted June 16, 2020. The copyright holder for this preprint (which was not certified by peer review) is the author/funder, who has granted bioRxiv a license to display the preprint in perpetuity. It is made available under aCC-BY-NC-ND 4.0 International license. 